\title{
FuPlex: A Full Duplex MAC for the Next Generation WLAN
}

\author{
(Invited Paper)
}

\author{
Qiao Qu, Bo Li, Mao Yang, Zhongjiang Yan, Xiaoya Zuo, and Qiaoyan Guan \\ School of Electronics and Information \\ Northwestern Polytechnical University, Xian, China \\ Email: quqiao21@163.com \\ \{libo.npu, yangmao, zhjyan, zuoxy\}@nwpu.edu.cn
}

\begin{abstract}
IEEE 802.11 wireless local area network (WLAN) has been increasingly developed over several decades. It requires four times throughput improvement in the next generation WLAN. Thus, researchers focus on the co-frequency co-time full duplex technology, which makes the devices transmit and receive packets simultaneously and theoretically doubles the throughput. Some existing works proposed several media access control (MAC) protocols on the assumption that all nodes have full duplex capability. However, it is more practicable that only AP possesses full duplex capability whereas STAs have no full duplex capability in the early stage of introducing full duplex technology into the next generation WLAN. In this paper, a simple and compatible full duplex MAC protocol named FuPlex is proposed. The design details of FuPlex, including primary access, secondary access and data transmission, are introduced. Simulation results show that FuPlex improves the throughput to $150 \%$ compared with legacy IEEE 802.11 MAC protocol.
\end{abstract}

\section{INTRODUCTION}

IEEE 802.11 wireless local area network (WLAN) is being deployed worldwide and lighting up our daily lives [1] [2]. The ever-increasing wireless traffic requires higher transmission capacity for the next generation WLAN. IEEE 802.11ax was approved in March 2014 to design a brand new amendment for the next generation WLAN [3]. IEEE 802.11ax requires four times throughput improvement in dense deployment scenarios compared with the current IEEE 802.11 WLAN. However, simply increasing the bandwidth for higher throughput will no longer be available since the spectrum resource is increasingly scarce. Therefore, new wireless communication technologies are needed to improve the spectrum efficiency in the next generation WLAN.

To achieve the objective mentioned above, co-frequency co-time full duplex technology has been propounded as a promising technology by enabling the devices to transmit and receive data packets at the same time and at the same frequency. It significantly improves the spectral efficiency of the wireless communication link, theoretically double. Most researchers paid attention to the physical layer implementation of full duplex technology, i.e. self-interference cancellation technologies [4]-[9]. However, full duplex technology cannot directly be adopted for the next generation WLAN without supportive medium access control (MAC) protocol. Therefore, it is necessary to study full duplex MAC protocol for the next generation WLAN.

There are some existing works focusing on the full duplex
MAC protocol design. Singh et. al. [10] proposed ContraFlow based on the traditional IEEE 802.11 MAC protocol, i.e. distributed coordination function (DCF). When any node receives a data packet for itself, it may also send another packet simultaneously. In other words, full duplex transmission enabled by ContraFlow only occurs when a node receives a data packet and has data packets for other nodes at the same time. FD-MAC [11] also adopts DCF as its channel access mechanism. Unlike ContraFlow, in FD-MAC whenever a node transmits a data packet, other nodes including the receiver could send a data packet to the sender, i.e. only the transmitting node could leverage full duplex technology to receive and transmit packets at the same time. Janus [12] is a centralized MAC protocol with AP scheduling. AP needs to firstly collect the interference and service information of STAs, and then broadcasts the scheduling information of the available full duplex transmission and the rate selection for STAs.

All the full duplex MAC protocols mentioned above are proposed on the same assumption that all the nodes, including AP and STAs, have full duplex capability. But this assumption is impracticable in the upcoming next generation WLAN because WLAN is considered and designed as a simple and low cost wireless network. Thus, in the early stage of the next generation WLAN, it is more likely that AP has full duplex capability but STAs have no full duplex capability. Therefore, the current full duplex MAC protocols may not suitable. Specifically, in ContraFlow, when STAs receive packets, they cannot send packets to establish full duplex transmission since STAs have no full duplex capability. In FD-MAC, when a STA sends a data packet at first, no full duplex transmission could occur since STAs are not able to receive a data packet while transmitting. Besides, because of the centralized character, Janus is difficult to be compatible with the legacy IEEE 802.11 WLAN. To the best of our knowledge this paper is the first work focusing on the scenario that only AP has full duplex capability and STAs have no full duplex capability. In this paper a simple and compatible full duplex MAC protocol named FuPlex for the next generation WLAN is proposed. In FuPlex, each node follows channel access mechanism in IEEE 802.11 DCF to ensure better compatibility with legacy WLAN, and the full duplex transmission is established and implemented during data transmission in IEEE 802.11 DCF. Thus, FuPlex not only leverages full duplex technology to achieve higher throughput, but also has good compatibility with legacy IEEE 802.11 WLAN. 
The contributions of this paper can be summarized as follows:

- This paper proposes a full duplex MAC protocol named FuPlex for the next generation WLAN on the assumption that only AP possesses full duplex capability, which adapts to the evolution of WLAN and has good compatibility.

- The simulation results demonstrate that FuPlex can improve throughput up to $150 \%$ compared with the IEEE 802.11 DCF in dense deployment scenarios.

The rest of this paper is organized as follows. In Section II, the design principles and overview of FuPlex are introduced. Then in Section III, the design details of FuPlex are described. Performance evaluation based on simulation is presented in Section IV to validate the performance of FuPlex. This paper is summarized in Section V.

\section{THE OVERVIEW OF FUPLEX}

The overview design of FuPlex is proposed in this section according to the demand of the next generation WLAN.

\section{A. Design Principles}

\section{1) Only APs possess full duplex capability}

Researchers express great interests in full duplex technology due to its advantage of theoretically doubling the spectrum efficiency. However, full duplex technology, on the other hand, will increase the complexity and cost of devices. Consequently, to implement full duplex technology only in AP is a relatively efficient and low-cost way in the upcoming next generation WLAN. Thus, the full duplex link should be an asymmetric link which consists of three nodes and two single links. As shown in Fig. 1, $\mathrm{STA}_{1}$ receives packets from AP, while $\mathrm{STA}_{2}$ transmits packets to AP. Thus, AP simultaneously receives packets from $\mathrm{STA}_{2}$ and transmits packets to $\mathrm{STA}_{1}$.

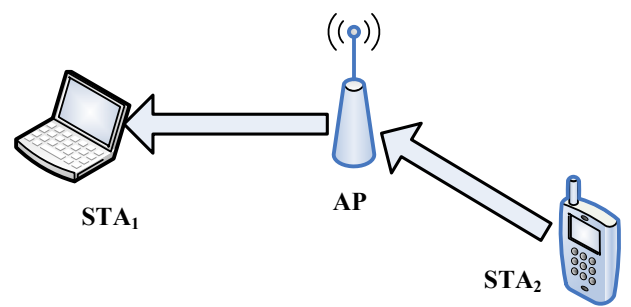

Fig. 1. Asymmetric full duplex link.

\section{2) Compatibility with legacy WLAN}

For the design of the next generation IEEE 802.11 standards, compatibility must be considered. Therefore, the full duplex MAC protocols for the next generation WLAN should follow the basic framework of IEEE 802.11 MAC protocol to possess better backward compatibility with IEEE $802.11 \mathrm{a} / \mathrm{b} / \mathrm{g} / \mathrm{n} / \mathrm{ac}$ [13] [14], e.g. the full duplex MAC protocols should be DCFbased.

\section{B. FuPlex Overview}

The overview design of the full duplex transmission for the next generation WLAN consists of two transmission procedures as shown in Fig. 2. The transmission procedure started at first refers to as the primary transmission procedure, and the later transmission procedure is denoted as the secondary transmission procedure. Primary transmission procedure consists of primary access, primary data transmission and primary acknowledgement (ACK) transmission, while secondary transmission procedure consists of secondary access, secondary data transmission and secondary ACK transmission. The definitions used in this paper is summarized in Table I.

TABLE I. DEFINITIONS IN THIS PAPER

\begin{tabular}{|l|l|}
\hline Definitions & Description \\
\hline Full Duplex Transmission & $\begin{array}{l}\text { A dual transmission using full duplex technology, } \\
\text { including primary transmission procedure and } \\
\text { secondary transmission procedure }\end{array}$ \\
\hline $\begin{array}{l}\text { Primary Transmission } \\
\text { Procedure }\end{array}$ & $\begin{array}{l}\text { The firstly starting transmission procedure, } \\
\text { including primary access, primary data } \\
\text { transmission and primary ACK transmission }\end{array}$ \\
\hline $\begin{array}{l}\text { Secondary Transmission } \\
\text { Procedure }\end{array}$ & $\begin{array}{l}\text { The later transmission procedure, including } \\
\text { secondary access, secondary data transmission } \\
\text { and secondary ACK transmission }\end{array}$ \\
\hline Primary Access & $\begin{array}{l}\text { The channel access mechanism in primary } \\
\text { transmission procedure }\end{array}$ \\
\hline Secondary Access & $\begin{array}{l}\text { The channel access mechanism in secondary } \\
\text { transmission procedure }\end{array}$ \\
\hline $\begin{array}{l}\text { Primary Data } \\
\text { Transmission }\end{array}$ & $\begin{array}{l}\text { The data transmission in primary transmission } \\
\text { procedure }\end{array}$ \\
\hline $\begin{array}{l}\text { Secondary Data } \\
\text { Transmission }\end{array}$ & $\begin{array}{l}\text { The data transmission in secondary transmission } \\
\text { procedure }\end{array}$ \\
\hline $\begin{array}{l}\text { Primary ACK } \\
\text { Transmission }\end{array}$ & $\begin{array}{l}\text { The ACK transmission in primary transmission } \\
\text { procedure }\end{array}$ \\
\hline $\begin{array}{l}\text { Secondary ACK } \\
\text { Transmission }\end{array}$ & $\begin{array}{l}\text { The ACK transmission in secondary transmission } \\
\text { procedure }\end{array}$ \\
\hline Primary Sender & The sender in primary transmission procedure \\
\hline Primary Receiver & The receiver in primary transmission procedure \\
\hline Secondary Sender & The sender in secondary transmission procedure \\
\hline Secondary Receiver & The receiver in secondary transmission procedure \\
\hline
\end{tabular}
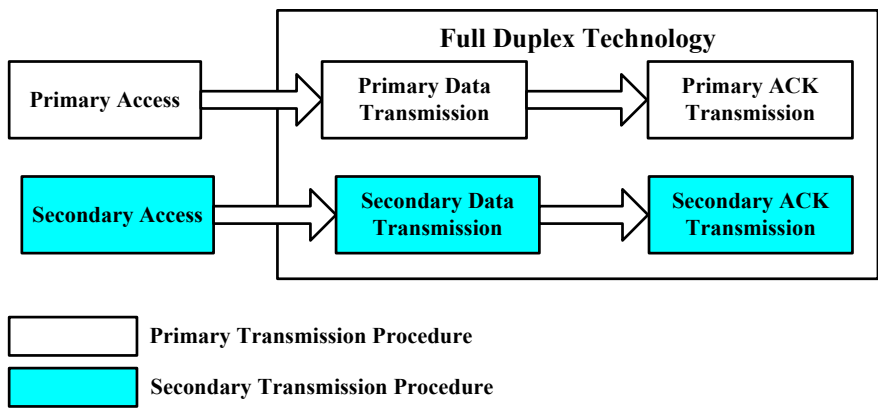

Fig. 2. The full duplex transmission in FuPlex.

\section{1) Primary transmission procedure}

In order to possess better compatibility with legacy IEEE 802.11 MAC protocol, primary access adopts the traditional IEEE 802.11 DCF for channel access mechanism. Since all nodes compete to access channel following IEEE 802.11 DCF, the primary transmission procedure could be initiated by AP, or by a STA. 


\section{2) Secondary transmission procedure}

After primary sender completes its primary access, secondary sender could access channel by secondary access mechanism. The secondary sender could be the AP in the current basic set service (BSS) if the primary sender is a STA, or a STA if the primary sender is the AP. Thus, there are totally two types of full duplex transmission: AP initiated full duplex transmission and STA initiated full duplex transmission. For the AP initiated full duplex transmission, the secondary sender should be a STA except for the primary receiver STA. On the other hand, for the STA initiated full duplex transmission, the secondary sender is the AP. Since the primary access employs IEEE 802.11 DCF to compete for channel resource, the main challenge for the full duplex MAC protocol design is how to implement secondary access.

\section{3) Interference in full duplex transmission}

It is important in full duplex transmission that the primary receiver successfully receives packets from the primary sender under the acceptable interference from the secondary sender, and the secondary receiver successfully receives packets from the secondary sender under the acceptable interference from the primary sender. For instance, in Fig. 3(a) the primary receiver $\mathrm{STA}_{2}$ not only receives a data packet from $\mathrm{AP}$, but also suffers from the interference from $\mathrm{STA}_{1}$. In this case, AP needs to adopt self-interference cancellation technology to cancel the self-interference. Similarly, in Fig. 3(b), when the full duplex transmission is initiated by $\mathrm{STA}_{2}, \mathrm{STA}_{1}$ could both receive a data packet from $\mathrm{AP}$ and suffer interference from $\mathrm{STA}_{2}$, and it is also necessary for AP to cancel the selfinterference. Therefore, interference in full duplex transmission should be considered carefully and thoughtfully. Since the selfinterference cancellation technology is fully studied [4]-[9], the main problem of full duplex transmission is to control the interference between two STAs involved in full duplex transmission so that primary transmission procedure and secondary transmission procedure can coexist.

\section{The Design Details of FuPleX}

This section provides the design details of FuPlex for the next generation WLAN according to the overall design consideration as described in Section II.

\section{A. MAC Procedure}

The procedure of FuPlex is illustrated in Fig. 4, including the procedure of AP initiated full duplex transmission in Fig. 4(a) and the procedure of STA initiated full duplex transmission in Fig. 4(b):

1) AP and STAs detect the channel according to the carrier sensing procedure defined in IEEE 802.11 DCF. After the channel has been idle for distributed inter-frame space (DIFS), AP and STAs start backoff procedure obeying the rules given in IEEE 802.11 DCF.

2) When a node, i.e. primary sender, completes the backoff procedure, it sends a request to send (RTS) packet to start primary transmission procedure, and then the primary receiver replies a clear to send (CTS) packet. In addition, nodes in the same BSS could measure the channel state information (CSI) during the RTS/CTS exchange.

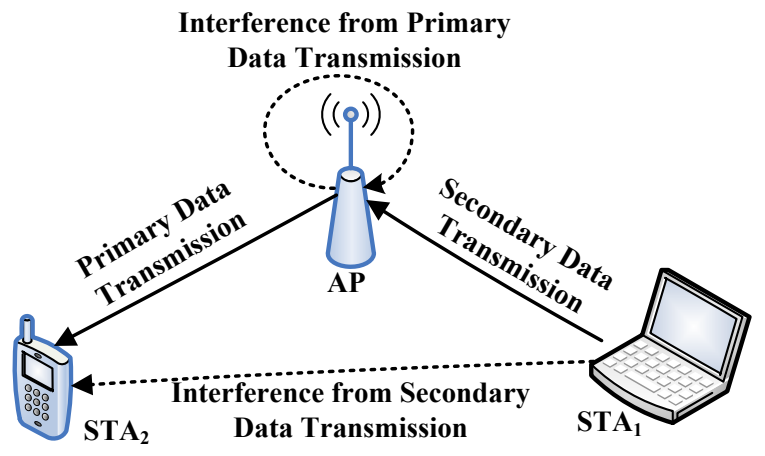

(a) Interference in AP initiated full duplex transmission

Interference from Secondary

Data Transmission

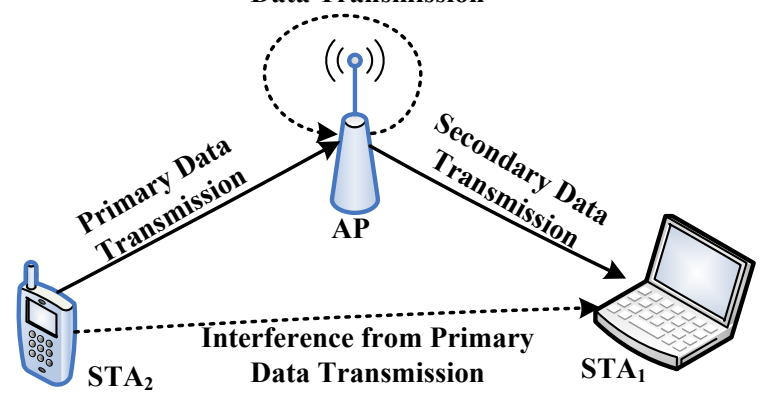

(b) Interference in STA initiated full duplex transmission

Fig. 3. Interference in two types of full duplex transmission.

3) After receiving the CTS packet, the primary sender firstly sends a data packet to the primary receiver. In AP initiated full duplex transmission, the STAs except for the primary receiver STA start secondary backoff procedure to compete for the secondary access, and the winner of the secondary access could directly transmit a data packet to the secondary receiver. While in STA initiated full duplex transmission, AP can use scheduling algorithm to select an appropriate STA as the secondary receiver according to buffer state information and historical transmission information of STAs. Moreover, the secondary sender has to receive the preamble of the data packet sent in the primary data transmission to acquire its duration.

4) The secondary sender needs to adjust (e.g. add some padding) its data transmission duration to make sure that both primary data transmission and secondary data transmission complete at the same time. Then the primary receiver and the secondary receiver simultaneously reply ACK to their senders. Thus, we highlight that full duplex technology is used both in data transmission period and ACK transmission period.

\section{B. Primary access}

In order to ensure better compatibility with legacy IEEE 802.11 WLAN, IEEE 802.11 DCF is employed as primary access mechanism for the FuPlex nodes, i.e. the nodes adopting FuPlex protocol. In other words, both legacy nodes and FuPlex nodes use the same rules to compete for channel resource. Thus, the fairness between FuPlex nodes and legacy nodes is guaranteed. In fact the primary transmission procedure and the secondary transmission procedure are independent from 


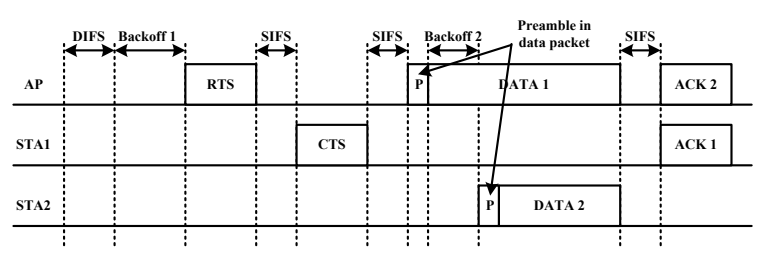

(a) AP initiated full duplex transmission

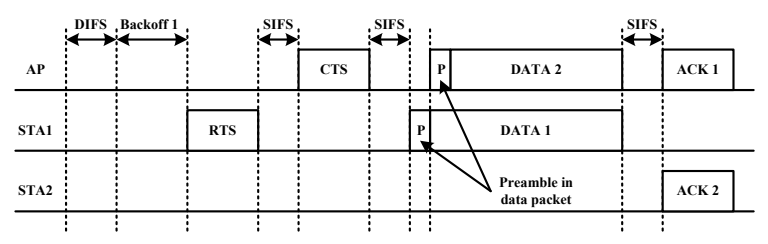

(b) STA initiated full duplex transmission

Fig. 4. The procedure of FuPlex.

the STAs' view except for the interference effect. The selfinterference cancellation technology is able to cancel the selfinterference existing in AP when it transmits packet and receives packet simultaneously, while CSI measurement during primary access is adopted to control interference between STAs.

In AP initiated full duplex transmission, STAs could measure the CSI during the procedure of RTS/CTS exchange as illustrated in Fig. 5(a). When $\mathrm{STA}_{2}$ receives the RTS packet from AP, it measures the receiving power $P_{R T S}$ and piggybacks $P_{R T S}$ on the CTS packet. Thus, once $\mathrm{STA}_{2}$ sends the CTS packet, the other STAs could measure the receiving power $P_{C T S}$ and obtain the piggybacked $P_{R T S} . P_{R T S}$ indicates the receiving power of $\mathrm{STA}_{2}$ for those packets sent by AP. $P_{C T S}$ indicates both the receiving power of $\mathrm{STA}_{2}$ for those packets sent by $\mathrm{STA}_{1}$ and the receiving power of $\mathrm{STA}_{1}$ for those packets sent by $\mathrm{STA}_{2}$ on the assumption that CSI is reversible [15] [16]. Therefore, $\mathrm{STA}_{1}$ could estimate the signal to interference and noise ratio (SINR) of DATA1 received by $\mathrm{STA}_{2}$ if $\mathrm{STA}_{1}$ sends DATA2 to AP simultaneously as shown in Fig. 5(b), and the estimated SINR is calculated as

$$
S I N R_{e}=\frac{P_{R T S}}{\left(P_{C T S}+N\right)}
$$

where $N$ is the power of noise. We define $S I N R_{T}$ as the minimal SINR value for primary receiver to successfully receive data packet. The STAs are allowed to perform secondary access if and only if the estimated $S I N R_{e}$ is equal to or larger than $S I N R_{T}$. In summary, when a STA receives a CTS packet, it checks the receiver address of the CTS packet. If the receiver of the CTS packet is the AP of the current BSS, the STA measures the receiving power $P_{C T S}$ and obtains the $P_{R T S}$ piggybacked in CTS, and then estimates the $S I N R_{e}$. If the estimated $S I N R_{e}$ is not less than $S I N R_{T}$, the STA could perform secondary access.

In STA initiated full duplex transmission, AP can use scheduling algorithm to select an appropriate STA as the secondary receiver. Thus, there is no extra CSI measurement procedure in primary access. In other words, the primary access in STA initiated full duplex transmission is as same as the channel access in IEEE 802.11 DCF.

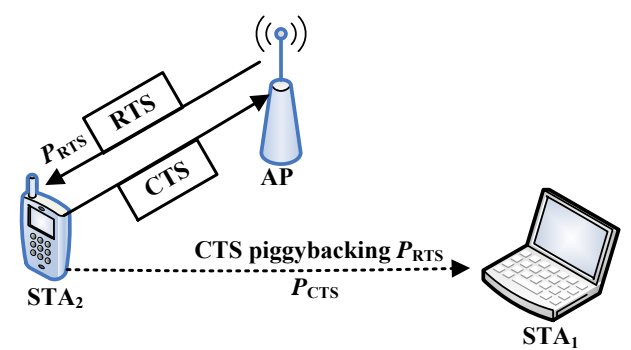

(a) CSI measurement in AP initiated full duplex transmission

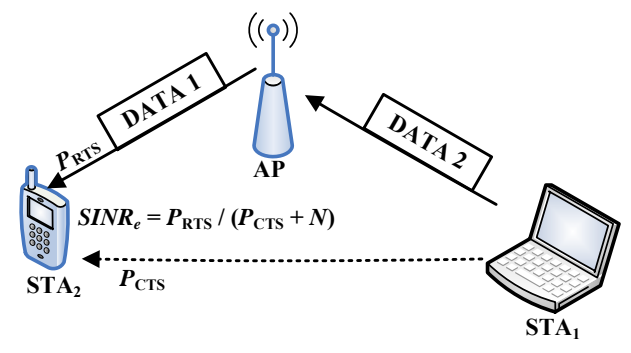

(b) Estimation of $S I N R_{e}$

Fig. 5. The procedure of CSI measurement and the estimation of $S I N R_{e}$.

\section{Secondary access}

Secondary backoff mechanism is employed for secondary access in AP initiated full duplex transmission. When AP sends data packet to the primary receiver STA, the other STAs except for the primary receiver STA need to receive the preamble of data packet sent by AP to get the data transmission duration from the L-SIG field [13]. Those STAs whose estimated $S I N R_{e}$ are not less than $S I N R_{T}$ start their secondary backoff procedures. To lower the possible interference to primary data transmission, the STAs with higher estimated $S I N R_{e}$ should have higher priority for secondary access. Thus, it is assumed that contention window in secondary access (denoted as $C W_{S}$ ) and $1 / S I N R_{e}$ is in linear relationship [17]. The STAs performing secondary backoff calculate the $C W_{S}$ value by Eq. (2) in [18]

$$
\frac{1}{S I N R_{e}}: \frac{1}{S I N R_{T}}=C W_{S}: C W_{S-M a x}
$$

where $C W_{S-M a x}$ is the maximal value of $C W_{S}$. After that, these STAs randomly choose a backoff counter from $\left[0, C W_{S}\right]$, and then start secondary backoff procedure respectively. Once a STA completes backoff process and sends a data packet to the AP, the other STAs sense the increase of power on the channel and determine that the channel is busy until the full duplex transmission completes. An example of secondary backoff procedure is illustrated in Fig. 6 .

In STA initiated full duplex transmission, since AP has all the downlink data packets to STAs, it is easy for AP to select a STA as the secondary receiver. Thus, centralized scheduling in AP is adopted as the secondary access mechanism. At first AP randomly chooses a STA as the secondary receiver and then records the ACK information. If AP receives ACK from secondary receiver STA, it indicates that this STA can establish full duplex transmission with the current primary sender STA together, and these two STAs are named as coupling STAs for 


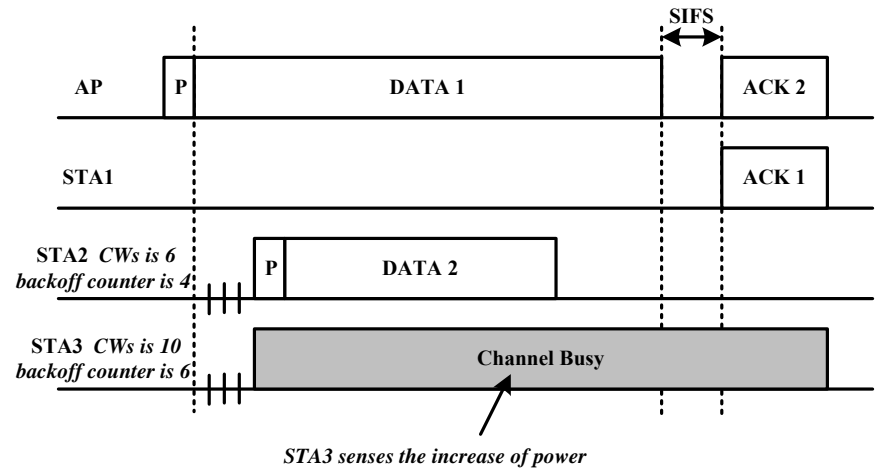

Fig. 6. When AP sends DATA1 to $\mathrm{STA}_{1}, \mathrm{STA}_{2}$ and $\mathrm{STA}_{3}$ need to receive the preamble of DATA1 to get the transmission duration of DATA1. The estimated $S I N R_{e}$ of $\mathrm{STA}_{2}$ and $\mathrm{STA}_{3}$ are both higher than $S I N R_{T}$, and then $\mathrm{STA}_{2}$ and $\mathrm{STA}_{3}$ start secondary backoff procedure independently. According to Eq. (2), $C W_{S}$ of $\mathrm{STA}_{2}$ and $\mathrm{STA}_{3}$ are 6 and 10 respectively. Then $\mathrm{STA}_{2}$ selects 4 as the backoff counter, and $\mathrm{STA}_{3}$ selects 6 as the backoff counter. Therefore, $\mathrm{STA}_{2}$ completes backoff process after four time slots, and sends DATA2 to AP Meanwhile, $\mathrm{STA}_{3}$ senses the increase of power on the channel and realizes that other STAs have started secondary data transmission. Thus, $\mathrm{STA}_{3}$ determines that the channel is busy until the full duplex transmission completes.

full duplex transmission. Thus, AP could use the information of coupling STAs for more efficient scheduling.

\section{Data transmission}

After receiving the preamble of the data packet in the primary data transmission, the secondary sender could get the transmission duration of the data packet. The secondary sender needs to select a proper data packet to make sure that secondary data transmission should not complete later than the primary data transmission. However, if the secondary sender completes data transmission earlier than the primary sender does, the ACK timeout problem occurs in FuPlex due to the ACK mechanism in IEEE 802.11 DCF. In AP initiated full duplex transmission, if the secondary sender STA completes data transmission earlier than AP does, AP cannot reply ACK to secondary sender STA immediately since it is sending a data packet to primary receiver. And in STA initiated full duplex transmission, if AP completes data transmission earlier than the primary sender STA does, AP could not receive ACK from secondary receiver STA since it is receiving a data packet from primary sender STA. Thus, ACK timeout problem may happen in both AP initiated full duplex transmission and STA initiated full duplex transmission. For instance, the ACK timeout problem mentioned above is described in Fig. 7.

To solve the ACK timeout problem, secondary sender could add some padding in its data packet to make sure that the secondary data transmission and primary data transmission complete simultaneously. In addition, to take full advantage of full duplex technology and further reduce the signaling overhead, the primary receiver and the secondary receiver simultaneously reply ACK to their senders. Thus, full duplex technology is used both in data transmission and ACK transmission.

\section{Performance eValuation}

In order to evaluate the performance of proposed full duplex MAC protocol, a simulation platform is built based

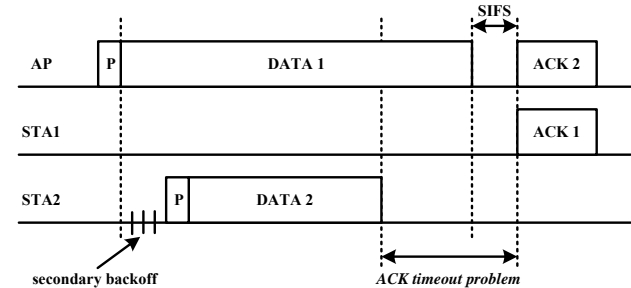

(a) AP firstly sends DATA1 to $\mathrm{STA}_{1}$. Then, after finishing the secondary backoff procedure, $\mathrm{STA}_{2}$ sends DATA2, and completes data transmission earlier than AP does. Since AP is sending DATA1, it cannot reply ACK to $\mathrm{STA}_{2}$ immediately. Therefore, $\mathrm{STA}_{2}$ considers that the reception of ACK is failed after a specific time.

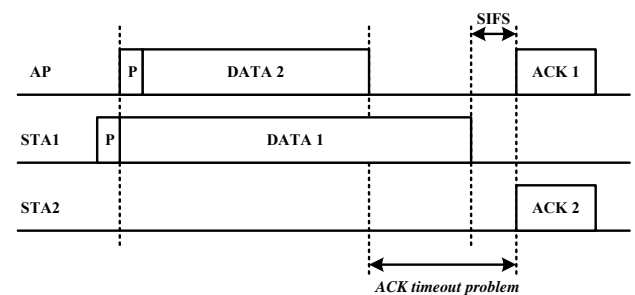

(b) $\mathrm{STA}_{1}$ sends DATA1 to AP at first. Then, AP sends DATA2 to $\mathrm{STA}_{2}$, and completes data transmission earlier than $\mathrm{STA}_{1}$ does. Since AP is receiving DATA1, it cannot receive ACK from $\mathrm{STA}_{2}$ at the same time. Thus, AP considers that the reception of ACK is failed after a specific time.

Fig. 7. The ACK timeout problem in FuPlex.

on NS2 [19]. The details of FuPlex as described above are implemented in this simulation platform. The simulation scenarios is in a single BSS, i.e. there are only one AP and some STAs around it. We assume that a packet could be successfully received only if the receiving SINR of this packet is not less than the $S I N R_{T}$. The parameters used in simulations are shown in Table II.

TABLE II. Simulation Parameters

\begin{tabular}{|c|c|}
\hline Parameters & Value in Simulation \\
\hline Preamble Duration & 20us \\
\hline Physical Rate & $6 \mathrm{Mbps}$ \\
\hline$S I N R_{T}$ & $3.16 \mathrm{~dB}$ \\
\hline$C W_{\min }$ & 15 \\
\hline$C W_{\max }$ & 1023 \\
\hline$C W_{S-M a x}$ & 15 \\
\hline DIFS & $34 \mathrm{us}$ \\
\hline SIFS & $16 \mathrm{us}$ \\
\hline Slot Time & 9us \\
\hline
\end{tabular}

\section{A. The number of STAs vs. Throughput}

Fig. 8 depicts throughput comparison of FuPlex and traditional IEEE 802.11 DCF under saturated traffic as the number of STAs increases. It shows that FuPlex always delivers a higher throughput than IEEE 802.11 DCF does since full duplex transmission is employed. FuPlex improves the throughput to $150 \%$ compared with the IEEE 802.11 DCF.

\section{B. The traffic rate vs. Throughput}

Fig. 9 illustrates the throughput for FuPlex compared with IEEE 802.11 DCF protocol in two scenarios with different 


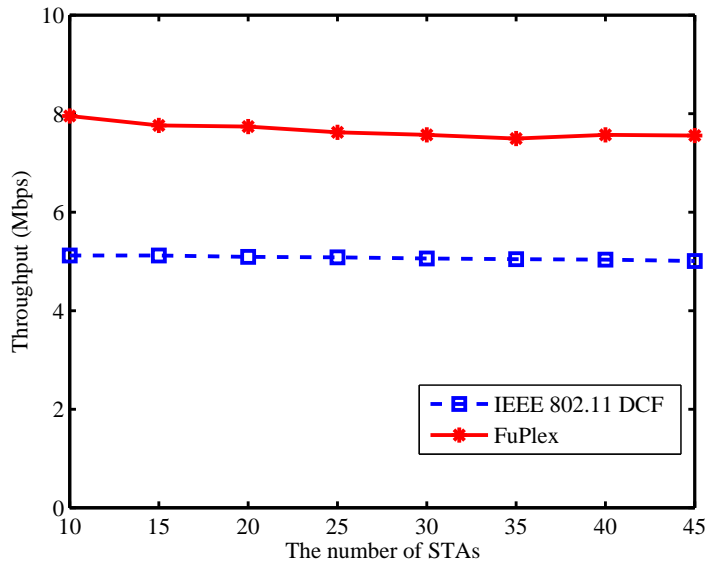

Fig. 8. The number of STAs vs. throughput under saturated traffic.

STA number. Constant bit rate (CBR) traffic is employed in our simulation scenarios. We can observe that the throughput with FuPlex is equal to that with IEEE 802.11 DCF in light traffic load, and is larger than that with IEEE 802.11 DCF in heavy traffic load since there are more opportunities for FuPlex to establish full duplex transmission under heavy traffic load. In a word, FuPlex is more suitable for heavy traffic load scenarios than IEEE 802.11 DCF, which evidently benefits the next generation WLAN.

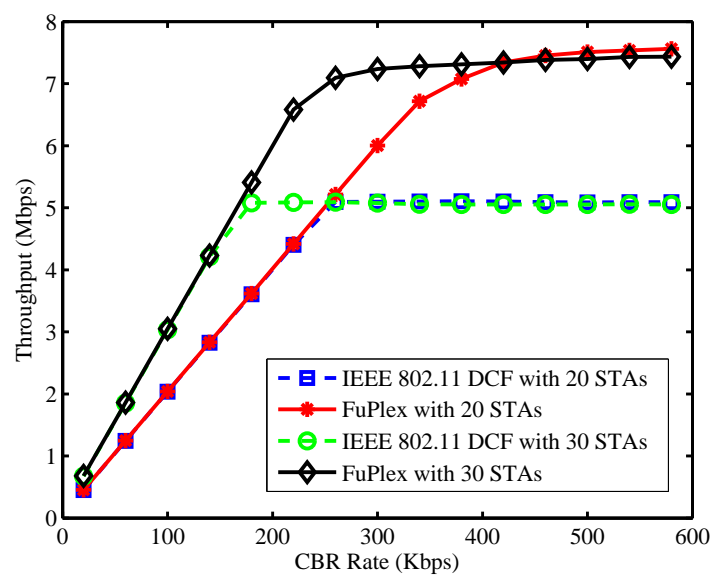

Fig. 9. The traffic rate vs. throughput.

\section{The number of STAs vs. Average packet delay}

In Fig. 10, the average packet delay is compared between FuPlex and IEEE 802.11 DCF, where the CBR traffic rate is $80 \mathrm{Kbps}$. The packet delay of FuPlex is always lower than that of IEEE 802.11 DCF since AP and STAs have more opportunities to transmit data packet in FuPlex. In addition, with the number of STAs increasing, the average packet delay of FuPlex increases relatively slowly compared with that of IEEE 802.11 DCF since more STAs means more opportunities to use full duplex transmission.

\section{CONClusion And Future Work}

This paper proposes a simple and compatible full duplex MAC protocol named FuPlex for the upcoming next generation

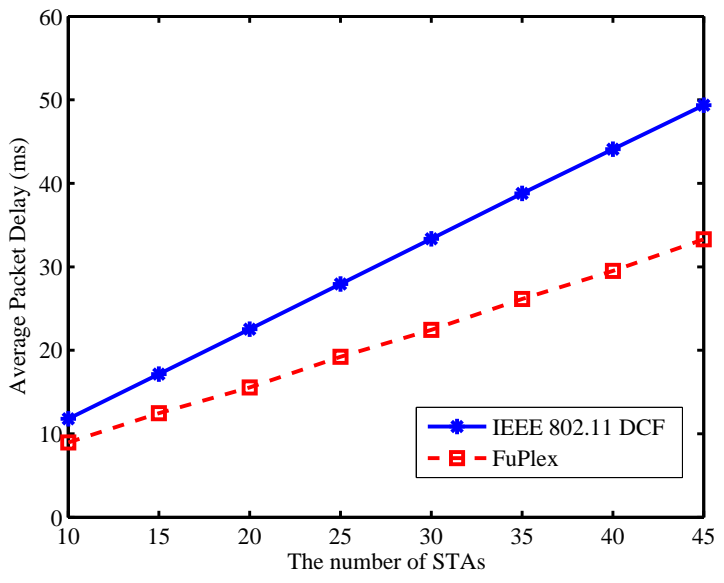

Fig. 10. The number of STAs vs. average packet delay.

WLAN on the assumption that only AP possesses full duplex capability. FuPlex not only improves the throughput by using full duplex technology, but also possesses better backward compatibility with legacy IEEE 802.11 MAC protocol. Simulation results show that the FuPlex protocol has advantage of higher throughput, lower packet latency and especially improving the throughput to $150 \%$ compared with that in IEEE 802.11 DCF in dense deployment scenarios. In the future work we will focus on the optimization algorithm of backoff contention window based on SINR.

\section{ACKNOWLEDGMENT}

This work is supported in part by the National Natural Science Foundations of CHINA (Grant No. 61271279, and 61201157), the National 863 plans project (Grant No. 2014AA01A707, and 2015AA011307), the National Science and Technology Major Project (Grant No. 2015ZX03002006), and the Fundamental Research Funds for the Central Universities (Grant No. 3102015ZY038, 3102015ZY039).

\section{REFERENCES}

[1] W. Sun, O. Lee, Y. Shin, S. Kim, C. Yang, H. Kim, and S. Choi, "Wifi could be much more," Communications Magazine, IEEE, vol. 52, no. 11, pp. 22-29, Nov 2014.

[2] R. Kudo, Y. Takatori, B. Hirantha Sithira Abeysekera, Y. Inoue, A. Murase, A. Yamada, H. Yasuda, and Y. Okumura, "An advanced wi-fi data service platform coupled with a cellular network for future wireless access," Communications Magazine, IEEE, vol. 52, no. 11, pp. 46-53, Nov 2014.

[3] D.-J. Deng, K.-C. Chen, and R.-S. Cheng, "Ieee 802.11ax: Next generation wireless local area networks," in Heterogeneous Networking for Quality, Reliability, Security and Robustness (QShine), 2014 10th International Conference on, Aug 2014, pp. 77-82.

[4] J. I. Choi, M. Jain, K. Srinivasan, P. Levis, and S. Katti, "Achieving single channel, full duplex wireless communication," in Proceedings of the Sixteenth Annual International Conference on Mobile Computing and Networking, ser. MobiCom '10. New York, NY, USA: ACM, 2010, pp. 1-12. [Online]. Available: http://doi.acm.org/10.1145/1859995.1859997

[5] S. S. Hong, J. Mehlman, and S. Katti, "Picasso: Flexible rf and spectrum slicing," SIGCOMM Comput. Commun. Rev., vol. 42, no. 4, pp. 37-48, Aug. 2012. [Online]. Available: http://doi.acm.org/10.1145/2377677.2377683 
[6] M. Duarte and A. Sabharwal, "Full-duplex wireless communications using off-the-shelf radios: Feasibility and first results," in Signals, Systems and Computers (ASILOMAR), 2010 Conference Record of the Forty Fourth Asilomar Conference on, Nov 2010, pp. 1558-1562.

[7] A. Sahai, "Wireless full-duplex: From practice to theory," Ph.D. dissertation, Univ. of Rice, Houston, Feb. 2014. [Online]. Available: https://scholarship.rice.edu/handle/1911/77524

[8] A. Sabharwal, P. Schniter, D. Guo, D. W. Bliss, S. Rangarajan, and R. Wichman, "In-band full-duplex wireless: Challenges and opportunities," CoRR, vol. abs/1311.0456, 2013. [Online]. Available: http://arxiv.org/abs/1311.0456

[9] D. Bharadia, E. McMilin, and S. Katti, "Full duplex radios," in Proceedings of the ACM SIGCOMM 2013 Conference on SIGCOMM, ser. SIGCOMM '13. New York, NY, USA: ACM, 2013, pp. 375-386. [Online]. Available: http://doi.acm.org/10.1145/2486001.2486033

[10] N. Singh, D. Gunawardena, A. Proutiere, B. Radunovic, H. Balan, and P. Key, "Efficient and fair mac for wireless networks with selfinterference cancellation," in Modeling and Optimization in Mobile, Ad Hoc and Wireless Networks (WiOpt), 2011 International Symposium on, May 2011, pp. 94-101.

[11] A. Sahai, G. Patel, and A. Sabharwal, "Pushing the limits of full-duplex: Design and real-time implementation," CoRR, vol. abs/1107.0607, 2011. [Online]. Available: http://arxiv.org/abs/1107.0607

[12] J. Y. Kim, O. Mashayekhi, H. Qu, M. Kaz, and P. Levis, "Janus: A novel mac protocol for full duplex radio," 2013. [Online]. Available: http://citeseerx.ist.psu.edu/viewdoc/summary?doi=10.1.1.381.1318

[13] "Ieee standard for information technology-telecommunications and information exchange between systems local and metropolitan area networks-specific requirements part 11: Wireless lan medium access control (mac) and physical layer (phy) specifications," IEEE Std 802.112012 (Revision of IEEE Std 802.11-2007), pp. 1-2793, March 2012.

[14] "Ieee standard for information technology- telecommunications and information exchange between systemslocal and metropolitan area networks- specific requirements-part 11: Wireless lan medium access control (mac) and physical layer (phy) specifications-amendment 4: Enhancements for very high throughput for operation in bands below 6 ghz." IEEE Std 802.11ac-2013 (Amendment to IEEE Std 802.11-2012, as amended by IEEE Std 802.11ae-2012, IEEE Std 802.11aa-2012, and IEEE Std 802.11ad-2012), pp. 1-425, Dec 2013.

[15] O. Punal, H. Escudero, and J. Gross, "Performance comparison of loading algorithms for $80 \mathrm{mhz}$ ieee 802.11 wlans," in Vehicular Technology Conference (VTC Spring), 2011 IEEE 73rd, May 2011, pp. 1-5.

[16] N. Mehta, A. Duel-Hallen, and W. Wang, "Enabling adaptive rate and relay selection for 802.11 mobile ad hoc networks," in Communications (ICC), 2012 IEEE International Conference on, June 2012, pp. 41504154.

[17] O. Branquinho, N. Reggiani, C. Correa, and D. Ferreira, "Wlan 802.11 mac anomaly mitigation using snr to control backoff contention window," in Microwave and Optoelectronics, 2005 SBMO/IEEE MTT-S International Conference on, July 2005, pp. 590-593.

[18] H. Kwon, S. Kim, and B. G. Lee, "Opportunistic multi-channel csma protocol for ofdma systems," Wireless Communications, IEEE Transactions on, vol. 9, no. 5, pp. 1552-1557, May 2010.

[19] The network simulator - ns2. [Online]. Available: http://www.isi.edu/nsnam/ns/ 